Post-print of:

Applied Optics, 55, 23, 6436-6442 (2016) doi:10.1364/AO.55.006436

Published 9 August 2016

\title{
GRASSP: A Spectrograph for the Study of Transient Luminous Events
}

\author{
María Passas ${ }^{1}{ }^{*}$, Justo Sánchez ${ }^{1}$, Ernesto Sánchez-Blanco ${ }^{2}$, Alejandro Luque ${ }^{1}$, And \\ FRANCISCO J. GORDILLO-VÁZQUEZ ${ }^{1}$
}

${ }^{1}$ Instituto de Astrofísica de Andalucía (IAA), CSIC, PO Box 3004, 18080 Granada, Spain

${ }^{2}$ Optical Development, Consultant, Avenida Pablo Iglesias 7 local 5, 41008 Sevilla, Spain

*Corresponding author: passasv@iaa.es

Compiled July 29, 2016

\begin{abstract}
We present the main parameters, design features and optical characterization of GRASSP, a ground-based spectrographic system intended for the analysis of the spectroscopic signature of transient luminous events (TLEs) occurring in the mesosphere of the Earth. It has been designed to measure the spectra of the light emitted from TLEs with a mean spectral resolution of $0.235 \mathrm{~nm}$ and $0.07 \mathrm{~nm} / \mathrm{px}$ dispersion in the wavelength range between 700 and $800 \mathrm{~nm}$. ๑) 2016 Optical Society of America
\end{abstract}

OCIS codes: (120.1880) Detection (120.6200) Spectrometers and spectroscopic instrumentation

http://dx.doi.org/10.1364/ao.XX.XXXXXX

\section{INTRODUCTION}

Transient Luminous Events (TLEs) are brief optical and electrical phenomena occurring in the upper regions of the Earth atmosphere after a sufficiently intense lightning stroke. They appear in several shapes and dimensions, lasting from less than several $\mathrm{ms}$ to tens of $\mathrm{ms}$ and acquiring dimensions of up to 500 $\mathrm{km}$ width (case of elves) or 50-90 km height (case of sprites and giant blue jets) $[1,2]$. Although presently detected only in the Earth atmosphere, TLEs could appear in every planet showing atmospheric electrical activity [3-5].

The first and simultaneous spectroscopic campaigns of TLEs were carried out in the mid 1990s [6,7], soon after the discovery of TLEs in 1989 [8]. These initial campaigns provided preliminary results on the optical emissions of TLEs corresponding to the first positive system (FPS) of $\mathrm{N}_{2}\left(\mathrm{~B}^{3} \Pi_{g} \rightarrow \mathrm{A}^{3} \Sigma_{u}^{+}\right)$in the visible and near infrared (NIR) spectral range (540-840 nm) recorded at standard video rate (30 fps) and at low (between 9 and $6 \mathrm{~nm}$ ) spectral resolution.

More recently, in 2007, spectroscopic observations of sprite optical emissions between $640 \mathrm{~nm}$ and $820 \mathrm{~nm}$ provided information on the relative vibrational concentrations of the emitting electronic state $\mathrm{N}_{2}\left(\mathrm{~B}^{3} \Pi_{g}, \mathrm{v}\right)$ at different altitudes using higher video rate (300 fps) and higher spectral resolution ( $3 \mathrm{~nm}$ ) spectrographs [9] originally designed for aurora spectroscopy [10].

The above mentioned sporadic TLE spectroscopic campaigns identified some of the key optical emissions from sprites (a type of TLE) and were even able to quantify some of the vibrational concentrations of the emitting levels in reasonable agreement with model predictions [11-13]. However, the best spec- tral resolution achieved to date is $3 \mathrm{~nm}$ and it is not enough to spectrally resolve the different low-lying vibrational transitions of the FPS of $\mathrm{N}_{2}$.

This work focuses on the development of a medium-high spectral resolution spectrograph aimed at characterizing from ground the spectroscopic fingerprints of TLEs occurring in the mesosphere of the Earth.

We describe here the GRanada Sprite Spectrograph and Polarimeter (GRASSP), an instrument that integrates a $0.235 \mathrm{~nm}$ resolution spectrograph covering the spectral range between $700 \mathrm{~nm}$ and $800 \mathrm{~nm}$, which will be used for systematic TLE spectroscopic surveys in Europe as part of the ground support to the Atmospheric Space Interaction Monitor (ASIM) space mission of the European Space Agency (ESA) to be launched in 2017.

The goal of GRASSP is to probe the mesosphere under the influence of all sort of TLEs (not only sprites) and to provide a systematic characterization of the spectroscopic signature of TLEs. The spectral resolution of GRASSP will allow to resolve for the first time the vibrational bands of the FPS of $\mathrm{N}_{2}$ in the transient air plasma spectra generated by TLEs and to remotely determine the rotational (gas) temperature using spectroscopic methods that require a minimum spectral resolution between $0.1 \mathrm{~nm}$ and $0.5 \mathrm{~nm}[14,15]$.

The scientific requirements for our instrument are detailed in Table 1.

\section{EXPERIMENTAL DEVICE DESIGN}

GRASSP currently consists of a medium-high resolution spectrograph, aimed manually, but with remotely-controlled inter- 


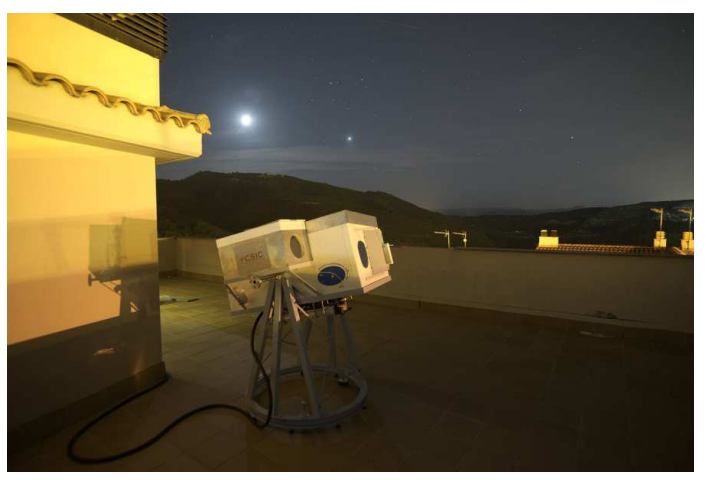

Fig. 1. GRASSP is currently installed in Castellgalí (Barcelona, Spain), at 275 meters above the mean sea level, $41.6768^{\circ} \mathrm{N}$ latitude and $1.8399^{\circ} \mathrm{E}$ longitude.

nal subsystems. Its housing is a result of a combination of two aluminum isothermal boxes -BOX1 and BOX2-, both installed on a manual altazimuth mount, presenting a frontal window with a known transmittance and equipped with manual and automatic blinds to protect the instrument from daylight (figure 1). BOX1 houses GRASSP spectrograph, while BOX2 will contain a polarimeter, which is currently under development, to measure and identify the direction and degree of polarization of TLEs optical emissions by calculating the 4 Stokes parameters from a single image.

Table 1. GRASSP Spectrograph Science Requirements

\begin{tabular}{lr}
\hline Wavelength range & $700 \mathrm{~nm}-800 \mathrm{~nm}$ \\
Mean spectral resolution & $0.1-0.5 \mathrm{~nm}$ \\
Mean resolving power & $>2500$ \\
\hline
\end{tabular}

\section{A. PC}

The GRASSP subsystems are managed by an Intel core i5 750 @ 2.67 GHz processor, with 2.99 GB RAM. It runs under Microsoft Windows XP Professional 2002 Service Pack 3 OS. We have chosen this operating system to avoid incompatibilities with the drivers of the devices connected. We have modified the electronics of the power on button of the PC to allow switching it on and off remotely.

The hardware components of GRASSP are represented in figure 2 .

\section{B. Static IP Switch}

The GRASSP instrument is controlled remotely thanks to a Conceptronic C100BRS4H broadband switch. It is powered by a 12 VDC supply of $500 \mathrm{~mA}$ and it has $4 \mathrm{LAN}$ and 1 WAN RJ-45 ports.

\section{Automaton}

The Schneider Electric OTB1E0DM9LP is a distributed module that switches on and off all subsystems through a software based on the Modbus protocol that we have implemented to remotely identify the inner PC status (on/off) by consulting its registers.

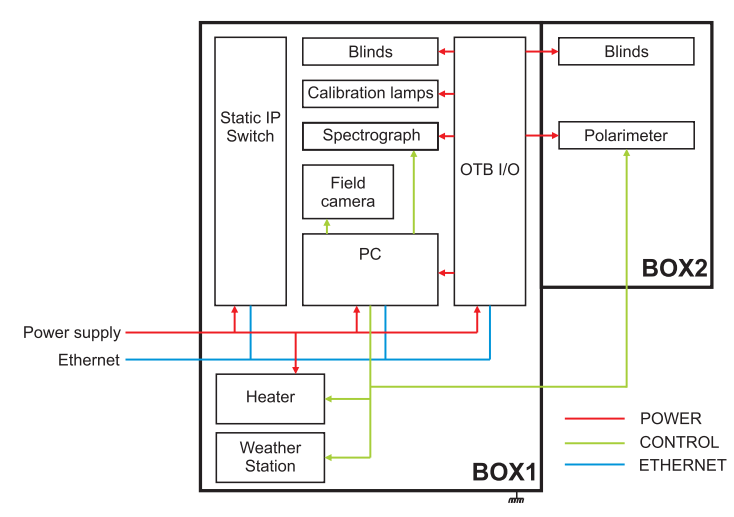

Fig. 2. GRASSP schematic. Box 1 contains GRASSP spectrograph; Box 2 will contain a polarimeter which is currently under development.

\section{Wide Field Camera}

The wide field camera records the night images and helps to discern the origin of the recorded spectrum. It consists of a Watec 1/2" Charge Coupled Device (CCD hereinafter): a WAT-902H2 ultimate with a $3005 \mathrm{VX} 4$ sensor low light monochrome camera of high sensitivity. Its CCD dimensions are $35.5 \times 40 \times 63 \mathrm{~mm}^{3}$ (width $x$ height $x$ length) and it weighs 98 grams. The number of total pixels of the CCD are $795(\mathrm{H}) \times 596(\mathrm{~V})$ with a unit cell size of $8.6 \mu \mathrm{m}(\mathrm{H}) \times 8.3 \mu \mathrm{m}(\mathrm{V})$. An auto iris function allows the camera to produce visible images in light levels as low as 0.0001 lux with a signal to noise ratio better than $50 \mathrm{~dB}$. Its field of view (FOV hereinafter) is $21.6(\mathrm{H}) \times 17.4(\mathrm{~V})$ degrees thanks to the \#F1.4 and $16 \mathrm{~mm}$ focal length lens it has attached. It has been focused to infinity, and records at a frame rate of 25 frames per second (fps) with a shutter speed mode control of $1 / 50$. A Honestech Trivid 4.0 HD analog to digital video capture system wires the Watec Camera through a BNC connector with the PC through a USB 2.0 host controller port. It supplies a color precision of 8 bits and a HD format support of $795(\mathrm{H}) \times 596(\mathrm{~V})$ pixels. The wide field camera specifications are summarized in Table 2.

Table 2. GRASSP Wide Field Camera

\begin{tabular}{lr}
\hline Wavelength range & $400 \mathrm{~nm}-1000 \mathrm{~nm}$ \\
Horizontal resolution & 600 lines \\
Signal to noise ratio & $50 \mathrm{~dB}$ \\
Automatic gain & $5-60 \mathrm{~dB}$ \\
Frame rate & $25 \mathrm{fps}$ \\
Field of view & $21.6^{\circ} \times 17.4^{\circ}$ \\
A/D converter & Honestech Trivid $4.0 \mathrm{HD}$ \\
Total pixels & $795 \times 596 \mathrm{px}(\mathrm{H} \times \mathrm{V})$ \\
\hline
\end{tabular}

\section{E. Audio Trigger System}

We have implemented an audio trigger system to start recording both the wide field camera and the spectrograph images 
simultaneously. This is possible thanks to a buffering system, which continuously stores the 30 last recorded frames of both the spectrograph and the wide field camera. When a change in brightness is detected in the wide field camera recording, an audio trigger is launched. A $3.5 \mathrm{~mm}$ male to male jack cable is connected to the headphones output and the microphone input of the computer, so the PC can receive the sound emitted and triggers the spectrograph recording, saving also all buffered images.

\section{F. Weather Station}

The PCE-FWS 20 meteorological station is a multi-functional device measuring $230 \mathrm{~mm} \times 150 \mathrm{~mm}$, which allows the accurate detection of temperature and relative humidity, both indoor and outdoor, among other weather conditions. Data can be date- and time-stamped to ensure that they can be analyzed effectively long after being collected.

\section{G. Thermal System}

An external intercooler and a heater are installed to keep the temperature inside GRASSP housing between 20 and 30 degrees Celsius. Both are painted in black to minimize their albedo and they are controlled by two relays activated by two different thermostats.

\section{GRASSP SPECTROGRAPH}

The spectrograph follows a classical collimator/camera arrangement with an intermediate collimated beam. The optical configuration of the GRASSP spectrograph is shown in Figure 3. The reflective ruled grating is placed at the pupil position in the collimated beam. The pupil diameter is $39.7 \mathrm{~mm}$ on the grating position. The collimator to camera angle is $38^{\circ}$. This geometry defines with the grating grooves the maximum angular dispersion. As this geometry has different angle of incidence and diffraction on the grating there is a magnification in the slit image (anamorphic factor for a non-Littrow geometry) as we move on the detector. The collimator and camera are off the self optics, with nominal $\mathrm{F}$ numbers faster than the nominal beam to avoid any vignetting.

The optics light path is as follows: the light is gathered with a $50 \mathrm{~mm}$ diameter aperture telescope with $170 \mathrm{~mm}$ effective focal length. Thus an F 3.4 beam is provided in the focal plane. Just before the focal plane, a field lens (focal length $190 \mathrm{~mm}$ ) places the pupil within the collimator to avoid vignetting. This field lens defines an observed FOV of $0.03^{\circ} \times 6^{\circ}$.

We developed an entrance slit of $31.47 \mathrm{~mm}$ length and variable width ranging between 0.08 and $0.10 \mathrm{~mm}$ by positioning two blades within two aluminum holders, parallel and vertical one over the other, leaving a gap between them. We used a calibrated feeler gauge of $0.10 \mathrm{~mm}$ to set the gap in first instance, and then we shortened the slit by manually tightening the upper blade with two micrometric screws. We oriented the entrance slit parallel to horizon to optimize the likelihood of TLE detection.

The f/2.8 and $135 \mathrm{~mm}$ focal length collimator provides a collimated beam and pupil that will reach the dispersive element. This is a 1440 grooves $/ \mathrm{mm}$ and $70 \times 70 \mathrm{~mm}$ aperture diffraction grating, with a blaze angle of $26.45^{\circ}$ at $540 \mathrm{~nm}$. The camera lens is a $50 \mathrm{~mm}$ focal length optic with a $\mathrm{f} / 1.7$.

Finally the detector system is a commercial monochrome intensified CCD L'Heritier LH 1300 ULL XVGA camera that provides the spectral pattern. The CCD is a Sony ICX285AL inter-

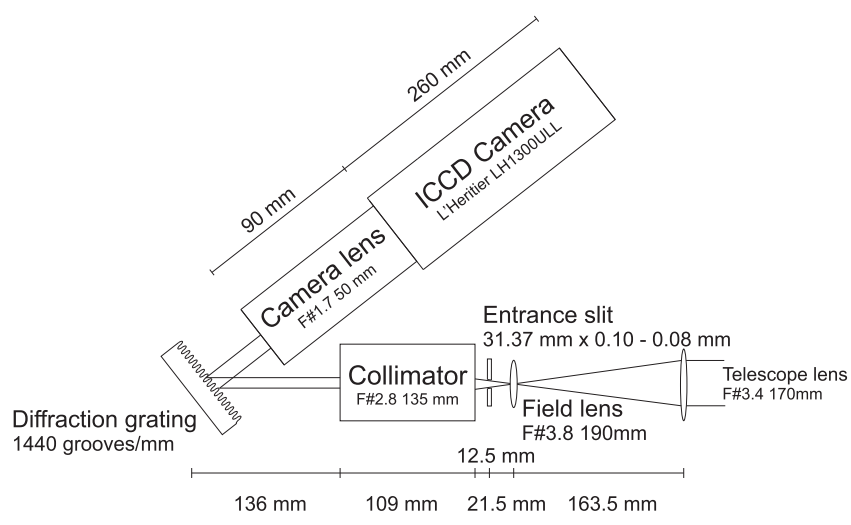

Fig. 3. Schematic of the GRASSP iCCD imaging spectrograph

line solid-state image sensor of $1360(\mathrm{H}) \times 1024(\mathrm{~V})$ pixels and $17.7 \mu \mathrm{m} / \mathrm{px}$. The streak tube of the image intensifier is a Photonis Super Gen S25 photocathode (with spectral response up to $950 \mathrm{~nm}$ ) equipped with a P20 phosphor, with a decay (persistence) time of tens of ms [16]. It is powered at $40 \mathrm{~W}$ maximum. The Super Gen S25 sensitivity reaches its maximum value in the $700-800 \mathrm{~nm}$ spectral range. In particular it varies between 65.7 and $67.7 \mathrm{~mA} / \mathrm{W}$ for wavelengths between 700 and $800 \mathrm{~nm}$, peaking at $750 \mathrm{~nm}$. Its quantum efficiency ranges between 11.6 $\%$ at $700 \mathrm{~nm}$ and $10.2 \%$ at $800 \mathrm{~nm}$. The iCCD is cooled by a Peltier system controlled by the PC through a RS232 connector. All different features of the camera are remotely controlled by the inner PC through a USB connector according to the instructions commanded by a Matlab software we have developed for that purpose.

The gain of this camera is manually adjustable in three different stages (figure 4): the exposure time stage, the photocathode intensifier tube gain (TG) stage and the electronic gain stage. First, we set an exposure time from $10 \mu$ s to $40 \mathrm{~ms}$, being this parameter the time during which the CCD collects photons thanks to an electronic shutter. This concept is different from the frame rate, which is always $25 \mathrm{fps}$ ( $40 \mathrm{~ms})$. When setting the exposure time to a value under $40 \mathrm{~ms}$, there is a loss of information corresponding to the period that the CCD is not collecting any photons. This is the reason we set the exposure time to the maximum value $(40 \mathrm{~ms})$ : as TLEs last few $\mathrm{ms}$, this exposure time guarantees the complete recording of the optical event. Once the exposure time is set, we can also increase the gain of the photocathode intensifier tube from $0 \mathrm{~dB}$ to $60 \mathrm{~dB}$. The final stage consists of an electronic gain from $0 \mathrm{~dB}$ to $30 \mathrm{~dB}$, obtaining a maximum intensification of $90 \mathrm{~dB}$ besides the selected exposure time.

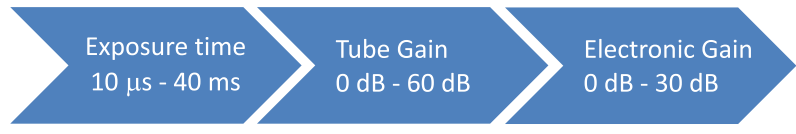

Fig. 4. L'Heritier camera gain stages

The L'Heritier iCCD output is digitized through a Blackmagic Design Decklink Mini Recorder analog to digital board. We selected the HD format $1080(\mathrm{~V}) \times 1920(\mathrm{H})$ pixels, although other modes are available. The chosen format supplies a 10-bit intensity precision, although we select only 8-bit precision due to software compatibilities. The computer interface is a PCI Express of 1 lane, compatible with 1, 4, 8 and 16 lane PCI Express 
slots. It is wired to the L'Heritier Camera through a SDI input.

The GRASSP spectrograph main features are summarized in Table 3.

\section{A. Spectrograph calibration}

The complete calibration of the spectroscopic system consists of three tasks: (1) a distortion correction, which straightens the raw curved spectrum; (2) a wavelength calibration, which assigns an absolute wavelength to each pixel of the iCCD; and (3) a flux calibration, which provides the spectral sensitivity of the spectrograph. Distortion correction and wavelength calibration are done whenever a spectrum is recorded; the instrument function is calculated just once.

\section{A.1. Distortion Correction and Wavelength Calibration}

Spectral images are commonly curved due to the aberration introduced by the optical elements of the instrument. To correct this undesirable effect, we used Argon and Kripton spectral lamps to record several images of each element spectrum with different gain levels. Once these images were combined forming a single synthetic frame with all spectral lines showing similar intensities, we trimmed it so all spectral lines appeared in every row of the spectrum (figure 5).

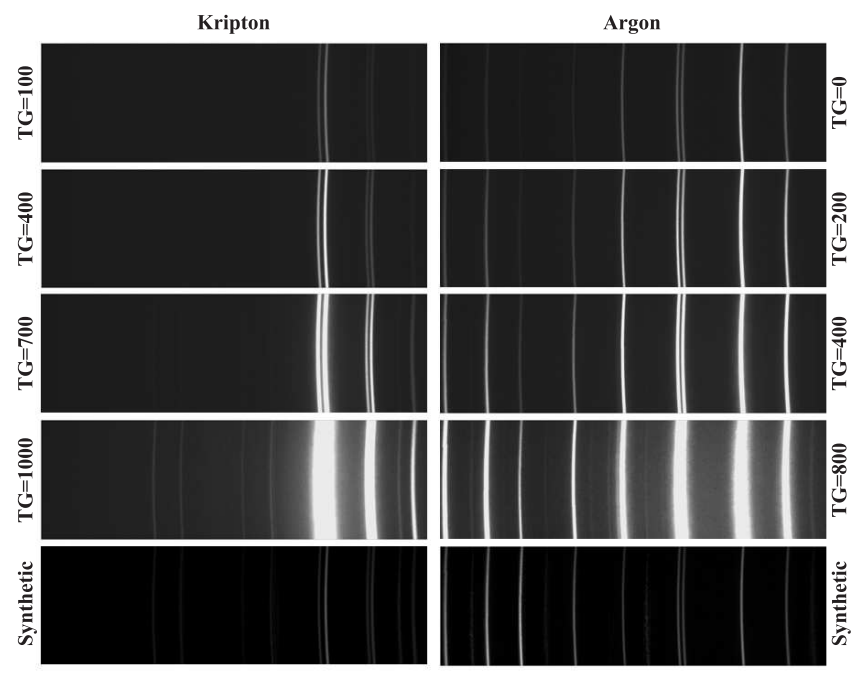

Fig. 5. Spectrum of Kripton (left) and Argon (right) recorded at different tube gain values. The last row shows the resulting synthetic spectra.

Then, we identified the corresponding wavelengths by comparing the distances between the spectral lines to the wellknown gaps that $\mathrm{Ar}$ and $\mathrm{Kr}$ spectra show [17]. Finally, to straighten the spectrum, we applied these wavelengths within a Matlab software we have implemented for that purpose. This code generates a calibration matrix which will be used hereinafter to correct the curvature of the spectra recorded with GRASSP associating every pixel coordinate in the iCCD to an absolute wavelength.

\section{A.2. Flux Calibration}

We have calculated the relative instrument function of the GRASSP spectrograph, using the true relative peak intensities of a well-known source as a function of wavelength. To do so, we obtained the spectral radiance of a halogen tungsten spectral

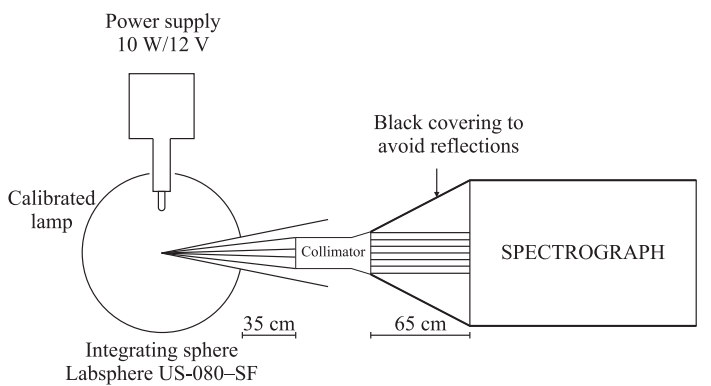

Fig. 6. GRASSP flux calibration setup

lamp (QTH) with GRASSP and we repeated the measurement with a calibrated Jobin Yvon - HORIBA FHR1000 spectrometer using the same calibration setup: we introduced the calibration lamp within a standard calibrated integrating sphere (Labsphere US-080-SF) of 8 " coated with Spectraflect $\mathrm{BaSO}_{4}$ base paint (see figure 6). The output light was collimated to reach both spectrographs, obtaining a spectrum. We covered part of the system (from the collimator to the spectrograph) with a black cloth to avoid undesirable reflections. We employed the same power supply, calibration lamp, integrating sphere, distances between optical elements and the same black covering. This way we minimized possible measuring errors. Equation 1 calculates the instrument function of GRASSP for row $i$ of the iCCD, according to the steps defined in [18].

$$
S(\lambda, i)=\frac{\text { spectrum }_{J O B I N-Y V O N}}{\text { Sprectrum }_{G R A S S P}(i)}
$$

The resulting relative conversion factor of the spectral sensitivity of the GRASSP system is calculated by dividing the spectral radiance curve of the commercial Jobin Yvon HORIBA FHR1000 spectrometer by each relative response of GRASSP spectrograph (one per row). Unfortunately, the iCCD shows a pattern that highlights the noise of the measure. To avoid this undesirable effect before calculating the conversion factor spectral sensitivity of the instrument, we smoothed the relative response of GRASSP spectrograph in every row by fitting the result by a tenth degree polynomial. Figure 7 shows this value for iCCD row number 26.

\section{B. Spectral Resolution}

We define the resolving power of the spectrograph as $R=\lambda / \delta \lambda$, with $\delta \lambda$ as the full width half maximum (FWHM) of a certain spectral emission line. These values are different in different areas of the iCCD, being worse in the edges. The mean calculated spectral resolution of GRASSP, in terms of its FWHM, and its mean resolving power are then $0.235 \mathrm{~nm}$ and 3309, respectively. The mean spectral dispersion of the GRASSP spectrograph is $0.07 \mathrm{~nm} / \mathrm{px}$. The current resolution element is contained in 3.3 pixels average (it changes across field, due to anamorphics and aberrations). It is worth highlighting that the spectral resolution could be increased a bit closer to the Nyquist limit (2 to 2.4 pixels). However, the narrower the slit is, the less photons reach the iCCD. So we had to find a compromise between obtaining the best spectral resolution with the maximum light intensity reaching the iCCD. We settled for $0.08 \mathrm{~mm}$ for the slit width as a good compromise between both requirements. 

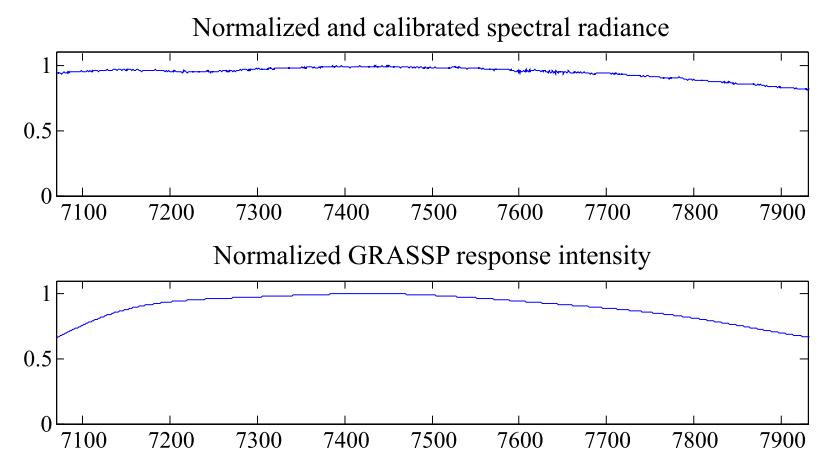

Conversion factor spectral sensitivity

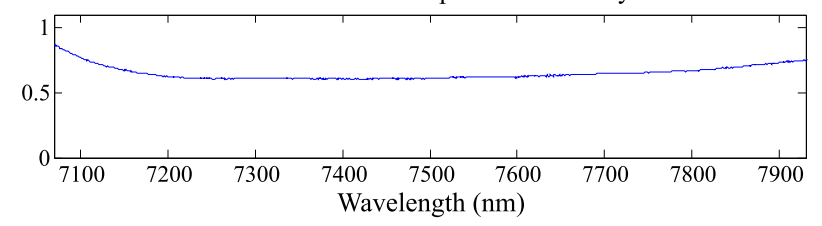

Fig. 7. Conversion factor spectral sensitivity of GRASSP instrument for row number 26.

\section{Spatial Resolution}

We calculated the spatial dispersion of the GRASSP spectrograph by measuring the spectrum of a full Moon. Viewed from Earth, the Moon subtends about $32 \pm 3$ minutes of arc. Since our spatial dispersion depends on the region of the iCCD, we measured the full Moon spectrum several times, and at different spatial positions of the iCCD. The spectrum of the full moon covered a mean value of $40 \pm 1$ pixels in the spatial axis. This way we found that the mean spatial dispersion of the GRASSP spectrograph is $48.0 \pm 4.5 \mathrm{arcsec} / \mathrm{px}$. We calculated the mean spatial resolution of GRASSP spectrograph by measuring the spectrum of a distant star, recorded in a new Moon night, in different positions of the iCCD and calculating its FWHM, being this value $3 \mathrm{px}$. The mean spatial resolution of GRASSP spectrograph is then $2.400 \pm 0.225 \mathrm{arcmin}$. This spatial resolution is worse than the theoretical expected value due to the effect of the photocathode stage over the focal plane of the camera.

\section{Stability}

The stability of a system defines the accuracy of the wavelength positions within the iCCD during one measurement period. It is important to stress that the inner temperature of GRASSP instrument is stable thanks to the cooling and temperature control systems. So if a wavelength shift is produced it is not due to temperature changes, but to the movements of the system during the observation night and, thus, it is not a fixed value.

We have calculated this parameter by wavelength calibrating the GRASSP spectrograph every hour while observing, and comparing the pixel position of every emission line within all calibrations. Figure 8 shows a mean shift of 3 pixels in 8 hours, whereas the curvature radii of the calibration spectral lines remain constant. The stability of the GRASSP spectrograph is better than 0.375 resolution elements per hour $(0.02625 \mathrm{~nm} /$ hour $)$.

We can shift the recorded spectrum according to the acquiring time to correct this effect by means of the calibration process we follow periodically in every observation night. Furthermore, the well-known wavelengths of the vibrational bands of the FPS of $\mathrm{N}_{2}$ can help to correct this shift.

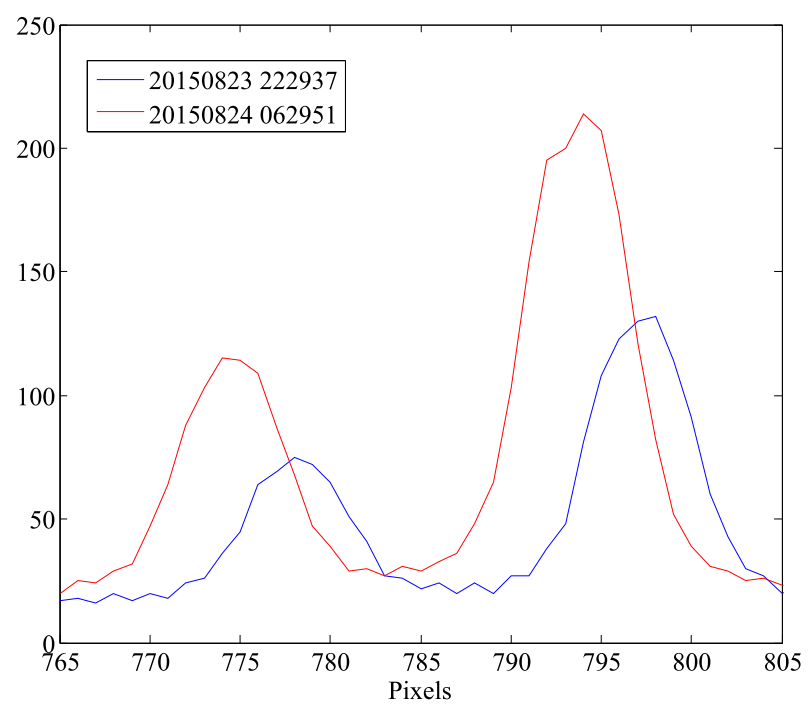

Fig. 8. Detail of calibration curves shift between pixels 765 and 805 in 8 hours. Blue line was measured at 22:29:37 the 23th August 2015. Red line was measured at 06:29:51 the 24th August 2015.

Table 3. GRASSP Spectrograph main features

Wavelength range

Mean spectral resolution

$700 \mathrm{~nm}-800 \mathrm{~nm}$

Mean resolving power

Mean spatial resolution

Stability

Configurable exposure time

Frame rate

Slit size

Field of view

A/D converter

Spectral image dimensions

\section{E. Spectrograph Reliability}

In order to test the instrument reliability, we compared the spectrum of the light emitted by a dry air arc lamp measured both with GRASSP and with a commercial Jobin Yvon HORIBA FHR1000 spectrometer. The air arc lamp geometry was cylindrical, $26 \mathrm{~cm}$ long and $0.5 \mathrm{~cm}$ width and fed by a DC source of $5 \mathrm{kV}$ and $10 \mathrm{~mA}$, at $0.2 \mathrm{mbar}$.

We set the configurable parameters of the FHR1000 spectrometer to provide a spectral resolution of $0.02 \mathrm{~nm}$, by selecting a dispersion grating of 1800 lines $/ \mathrm{mm}$, a slit of $24 \mu \mathrm{m}$ and a mean integration time of $1 \mathrm{~s}$, being the detector a CCD of $26 \mu \mathrm{m} / \mathrm{px}$. Then, we convolved the resulting spectrum with a gaussian curve of standard deviation of $0.11 \mathrm{~nm}$ and $0.18 \mathrm{~nm}$ to perform two synthetic spectra of $0.23 \mathrm{~nm}$ and $0.37 \mathrm{~nm}$ spectral resolution, respectively.

The GRASSP spectrograph was set to $40 \mathrm{~ms}$ exposure time and a tube gain of 1200 . Due to the setup of the measurement system, less tube gain values did not allow to discern all the 
spectral features of air between 700 and $800 \mathrm{~nm}$.

To compare both spectra we performed the following data reduction steps: bias subtraction, flat fielding, signal to noise ratio enhancement, iCCD inhomogeneities correction, background subtraction, distortion correction and wavelength calibration, flux calibration, and finally, combination of a set of images to extract the desired spectrum.

\section{E.1. Bias Subtraction}

Bias images are recorded without exposing the iCCD to any incident light. To eliminate bad pixel counts due to incident cosmic rays, we combined a group of ten bias images by means of the median value of each pixel $\left(\right.$ Bias $\left._{C}\right)$.

\section{E.2. Flat Fielding}

Flat images are recorded exposing the chip to a homogeneous light. To eliminate bad pixel counts due to incident cosmic rays, we combined a group of ten flat images by means of the median value of each pixel $\left(\right.$ Flat $\left._{c}\right)$. This resulting image will correct the differences between pixel sensitivities of the iCCD.

\section{E.3. Signal to Noise Ratio Enhancement}

We combined a group of ten spectrum images of the light emitted by the air lamp to enhance the signal to noise ratio. This combination consisted of calculating the median value of each pixel for each image, generating a synthetic image, $\left(\right.$ Spectrum $\left._{\mathcal{c}}\right)$.

\section{E.4. iCCD inhomogeneities correction}

Equation 2 corrects the iCCD inhomogeneities of the air lamp spectrum image.

$$
\operatorname{Air}_{c}=\frac{\text { Spectrum }_{\mathcal{C}}-\text { Bias }_{\mathcal{C}}}{\text { Flat }_{c}-\text { Bias }_{\mathcal{C}}}
$$

\section{E.5. Background subtraction}

The background emission is defined in astronomy as the incoming light from an apparently empty part of the night sky. We subtracted a combination of ten background images -recorded at $40 \mathrm{~ms}$ exposure time and a tube gain of 1000- to the signal area according to equation 3 .

$$
\operatorname{Air}_{b}=\text { Air }_{c}-\text { Background }_{c}
$$

\section{E.6. Distortion correction and wavelength calibration}

The resulting image from the steps above is curved due to the optical aberration of the system. To correct this undesirable effect, we applied the wavelength calibration matrix calculated previously in the distortion correction and wavelength calibration step: we straightened the spectrum of the air lamp and we assigned an absolute wavelength to every pixel of the iCCD. Figure 9 shows the resulting corrected image.
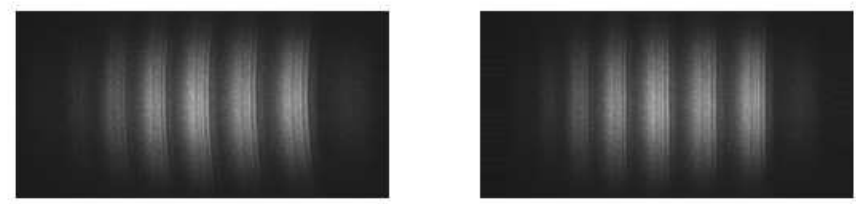

Fig. 9. Air lamp spectral images: curved (left) and straightened (right).

\section{E.7. Flux calibration}

To repair the undesired effects introduced by the spectroscopic system over the real image, we finally multiplied the resulting spectrum by the conversion factor of the instrument response function, previously calculated in equation 1 .

$$
\operatorname{Air}_{F I}=\operatorname{Air}_{b}(i) \cdot S(\lambda, i)
$$

\section{E.8. Resulting spectrum}

We integrated each column of the resulting air lamp spectrum image, measured with GRASSP. Figures 10 and 11 show the air lamp recorded spectra with both GRASSP and Jobin Yvon Horiba FHR1000 spectrometers.

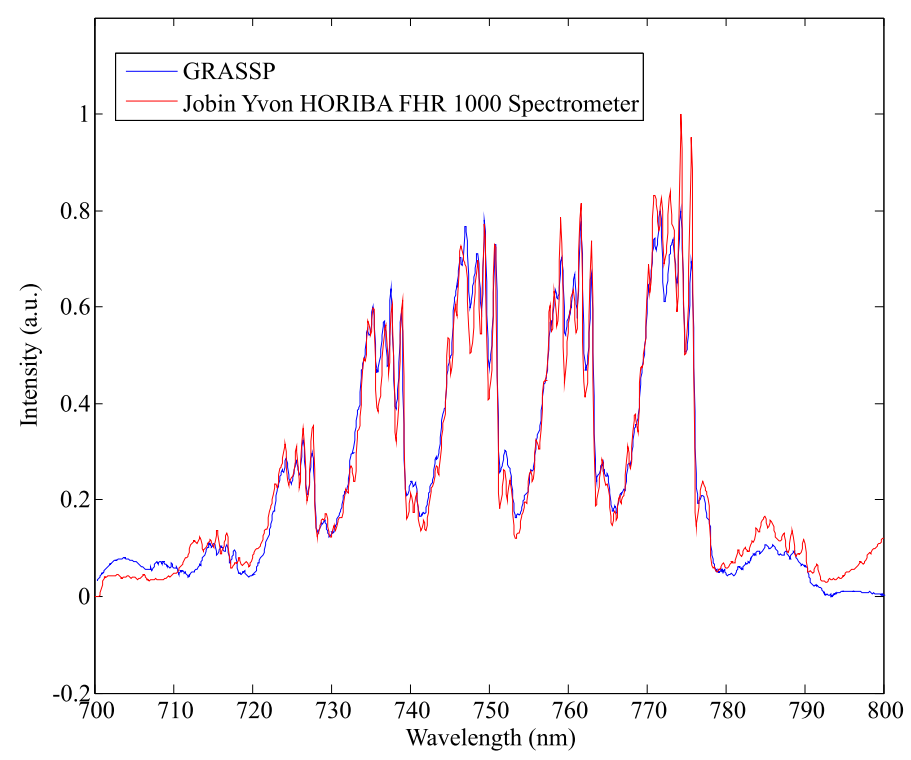

Fig. 10. Spectra of a dry air (0.2 mbar) lamp measured with a commercial Jobin Yvon Horiba FHR1000 spectrometer (red) at $0.36 \mathrm{~nm}$ spectral resolution and with GRASSP spectrograph (blue).

Although the GRASSP and Jobin Yvon Horiba FHR1000 measurements were taken according to a similar setup, there are several factors that may make the height of the peaks not to be coincident, such as the different ambient lighting during the measurements or variations in the times the lamp bulb was switched on before the measurements, which result in a different temperature of the air plasma. Note also that the tube gain values were different when recording the air spectrum -tube gain 1200- and the background -tube gain 1000-. Hence, the subtraction of the background may not be accurate enough resulting in a slight difference between both background estimations.

Additionally, the differences between the spectral resolution of both spectrographs might also have influence in these inequalities. The spectral resolution of the GRASSP spectrograph is not constant at all wavelengths and, on the other hand, we set two fixed wavelength resolutions for the Jobin Yvon Horiba FHR1000 spectrometer. Notice that the agreement of both spectra (FHR1000 and GRASSP) is better depending on the wavelength range we are observing and on the spectral resolution selected for the Jobin Yvon Horiba FHR1000 spectrometer.

By inspecting figures 10 and 11, one might be tempted to think that the mean spectral resolution is higher than $0.235 \mathrm{~nm}$, 


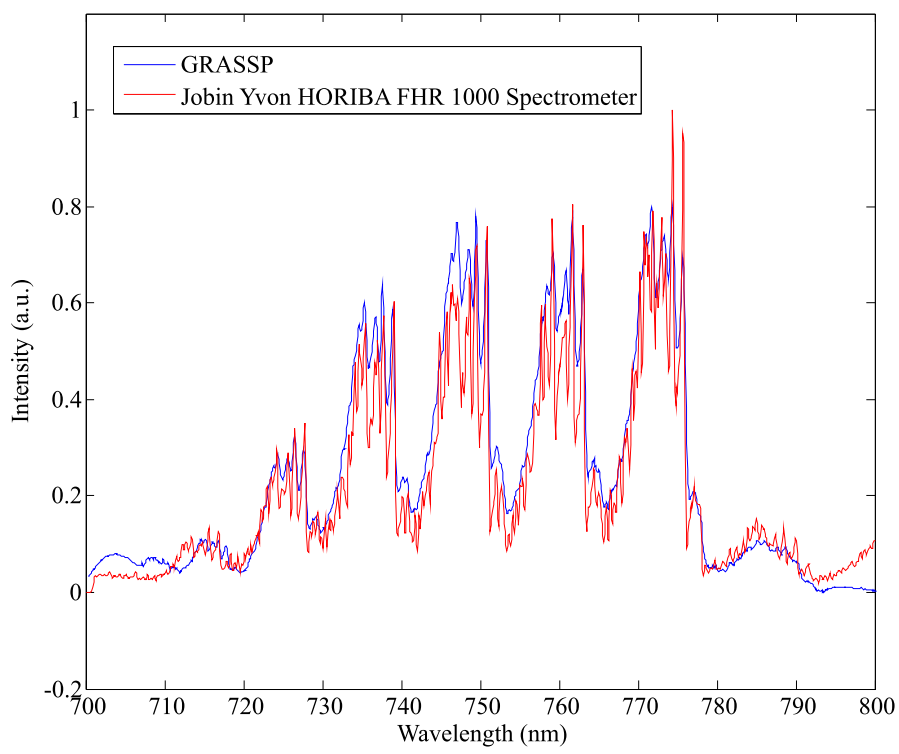

Fig. 11. Spectra of a dry air (0.2 mbar) lamp measured with a commercial Jobin Yvon Horiba FHR1000 spectrometer (red) at $0.23 \mathrm{~nm}$ spectral resolution and with GRASSP spectrograph (blue).

but it is not. We calculated this value by means of the FWHM of the spectral lines of the Argon and Kripton lamps at an optimal gain of the iCCD. When rising up the iCCD gain, this FWHM widens. So if we measure the air lamp spectrum with less iCCD gain, we will not see the lower peaks of the vibrational bands between 710 and $720 \mathrm{~nm}$ and between 780 and $790 \mathrm{~nm}$, but we will discern the spectral structure with higher spectral resolution between 720 and $780 \mathrm{~nm}$.

Besides, the reliability of GRASSP spectrograph decreases as we approach the edges of the iCCD.

Despite these differences, this comparison confirms the reliability of the wavelength and flux calibration of the GRASSP spectrograph between 715 and $790 \mathrm{~nm}$.

\section{CONCLUSION}

In this paper, we have described the design and development as well as the complete optical characterization of GRASSP, a new imaging spectrographic system with $0.235 \mathrm{~nm}$ spectral resolution. GRASSP is a spectrograph specifically designed to carry out systematic TLE spectroscopic surveys in Europe.

Thanks to its spectral resolution, GRASSP will resolve for the first time the rotational structure within the vibrational bands of the FPS of $\mathrm{N}_{2}$ in the transient air plasma spectra emitted by TLEs, thus allowing to remotely determine the rotational (gas) temperature. It is worth highlighting that the GRASSP spectrograph is the first instrument designed and developed specifically for automatic and systematic detection of TLE spectroscopic features, improving the best spectral resolution to date of former TLE spectrographs by up to ten times.

\section{FUNDING INFORMATION}

This work was supported by the Spanish Ministry of Science and Innovation, MINECO under projects ESP2013-48032-C5-5R, ESP2015-69909-C5-2-R and FIS2014-61774-EXP and by the EU through the FEDER program. AL acknowledges support by a Ramón y Cajal contract, code RYC-2011-07801.

\section{ACKNOWLEDGMENTS}

The authors gratefully acknowledge Dr. Isabel Tanarro for her invaluable assistance in measuring the calibrated lamp spectrum with the Jobin Yvon Horiba FHR1000 spectrometer under her supervision in the Instituto de Estructura de la Materia (IEM), CSIC, Madrid, Spain.

\section{REFERENCES}

1. T. Neubert, T. H. Allin, H. Stenbaek-Nielsen, and E. Blanc, "Sprites over Europe," Geophys. Res. Lett. 28, 3585 (2001).

2. V. P. Pasko, M. A. Stanley, J. D. Mathews, U. S. Inan, and T. G. Wood, "Electrical discharge from a thundercloud top to the lower ionosphere," Nature 416, 152-154 (2002).

3. Y. Yair, Y. Takahashi, R. Yaniv, U. Ebert, and Y. Goto, "A study of the possibility of sprites in the atmospheres of other planets," J. Geophys. Res. (Planets) 114(E13), E09,002 (2009). 0812.0258.

4. D. Dubrovin, A. Luque, F. J. Gordillo-Vázquez, Y. Yair, F. C. ParraRojas, U. Ebert, and C. Price, "Impact of lightning on the lower ionosphere of Saturn and possible generation of halos and sprites," Icarus 241, 313-328 (2014).

5. A. Luque, D. Dubrovin, F. J. Gordillo-Vázquez, U. Ebert, F. C. ParraRojas, Y. Yair, and C. Price, "Coupling between atmospheric layers in gaseous giant planets due to lightning-generated electromagnetic pulses," J. Geophys. Res.: Space Physics 119, 8705-8720 (2014).

6. S. B. Mende, R. L. Rairden, G. R. Swenson, and W. A. Lyons, "Sprite spectra; $N_{2} 1$ PG band identification," Geophys. Res. Lett. 22, 2633 (1995).

7. D. L. Hampton, M. J. Heavner, E. M. Wescott, and D. D. Sentman, "Optical spectral characteristics of sprites," Geophys. Res. Lett. 23, 89 (1996).

8. R. C. Franz, R. J. Nemzek, and J. R. Winckler, "Television Image of a Large Upward Electrical Discharge Above a Thunderstorm System," Science 249, 48 (1990).

9. T. Kanmae, H. C. Stenbaek-Nielsen, and M. G. McHarg, "Altitude resolved sprite spectra with $3 \mathrm{~ms}$ temporal resolution," Geophys. Res. Lett. 34, L07,810 (2007).

10. T. J. Hallinan, H. C. Stenbaek-Nielsen, and C. S. Deehr, "Enhanced Aurora," J. Geophys. Res. 90, 8461-8475 (1985).

11. F. J. Gordillo-Vázquez, A. Luque, and M. Simek, "Spectrum of sprite halos," J. Geophys. Res. (Space Phys) 116, A09,319 (2011).

12. A. Luque and F. J. Gordillo-Vázquez, "Modeling and analysis of $\mathrm{N}_{2}\left(\mathrm{~B}^{3} \Pi_{g}\right)$ and $\mathrm{N}_{2}\left(\mathrm{C}^{3} \Pi_{u}\right)$ vibrational distributions in sprites," J. Geophys. Res. (Space Phys) 116(A15), A02,306 (2011).

13. F. J. Gordillo-Vázquez, A. Luque, and M. Simek, "Near infrared and ultraviolet spectra of TLEs," J. Geophys. Res. (Space Phys) 117, A05,329 (2012).

14. M. Simek and S. DeBenedictis, "On the use of the numerical simulation of the first positive system of $\mathrm{N}_{2}$ : II. Fast $\mathrm{T}_{\text {rot }}$ Estimation from the Partially Resolved (3,0) Band," Plasma Chem. Plasma Proc. 15, 451-463 (1995).

15. F. C. Parra-Rojas, M. Passas, E. Carrasco, A. Luque, I. Tanarro, M. Simek, and F. J. Gordillo-Vázquez, "Spectroscopic diagnostics of laboratory air plasmas as a benchmark for spectral rotational (gas) temperature determination in TLEs," J. Geophys. Res. 118, 46494661 (2013).

16. P. Hoess and K. Fleder, "Time Integrated Phosphor Behavior in Gated Image Intensifier Tubes," Proc. SPIE 4128, 23-28 (2000).

17. A. Kramida, Yu. Ralchenko, J. Reader, and and NIST ASD Team, NIST Atomic Spectra Database (ver. 5.2), [Online]. Available: http://physics .nist.gov/asd [2015, September 22]. National Institute of Standards and Technology, Gaithersburg, MD. (2014).

18. U. Fantz, "Basics of plasma spectroscopy," Plasma Sources Science and Technology 15(4), 137-147 (2006). 